\title{
EFFECT OF THERMO-MECHANICAL AGING ON THE SURFACE ROUGHNESS AND MICRO-HARDNESS OF FOUR CAD/CAM MATERIALS
}

\author{
Rania A Amin *, Dalia A Saba ** and Mostafa A Abdellatif ***
}

\begin{abstract}
Aim of the study: The aim of this study was to evaluate the changes in surface roughness and micro-hardness of four CAD/CAM materials after thermo-mechanical aging.

Materials and methods: Four commercially available CAD/CAM materials were investigated in this study; two ceramic materials; CEREC Blocs, Celtra Duo, and two hybrid ceramics; CERASMART and VITA ENAMIC. The materials' blocks were cut into 5 disks each and were subjected to thermo-mechanical aging through 250,000 thermocycles in a mechanical chewing simulator. Surface roughness and micro-hardness were evaluated for each material pre and post thermo-mechanical aging using digital image processing technology and Vickers hardness testing machine, respectively. Results were statistically analyzed using one way analysis of variance (ANOVA), Bonferroni Post Hoc test and paired t test $(\mathrm{P} \leq 0.05)$.
\end{abstract}

Results: Regarding surface roughness; there was no significant difference between all investigated materials before thermo-mechanical aging. After aging, all materials showed insignificant difference between them and insignificant change between pre and post aging with exception of CERASMART that showed significant increase in surface roughness. Regarding surface micro-hardness, the tested materials showed significant difference pre aging in the ascending order of Cerasmart < Enamic < Cerec Blocks < Celtra Duo. But after aging, all materials showed insignificant difference in surface micro-hardness between them and insignificant change between before and after the test.

Conclusions: Within the limitations of the current study, it can be concluded that although within the clinical acceptable range, the surface roughness of Cerasmart was significantly affected by the thermo-mechanical aging. The composition is still the most important parameter affecting the surface roughness and micro-hardness of CAD/CAM ceramic and hybrid ceramic materials

KEY WORDS: CEREC, Celtra Duo, CERASMART, VITA ENAMIC, Aging, Chewing simulation

\footnotetext{
* Assistant Professor in Crowns and Bridges Department, Faculty of Dental Medicine, Al-Azhar University (Girls' branch), Egypt.

** Associate Professor of Dental Materials Science, Biomaterials Department, Faculty of Dentistry, Cairo University, Egypt.

*** Associate Professor of Dental Materials Science, Biomaterials Department, Faculty of Oral and Dental Medicine, Future University in Egypt, FUE.
} 


\section{INTRODUCTION}

Over the last three decades, computer-assisted design and computer-assisted manufacturing (CAD/ CAM) was considered the most actively used technology in dentistry being time saving, eliminating potential error sources and improving accuracy of indirect restorations. ${ }^{1,2}$ A wide range of materials were developed based on CAD/CAM technology, one of the recent and most preferable by clinicians is the monolithic blocks. Monolithic CAD/CAM blocks can overcome the chipping of low strength veneering layer of multilayered restorations. ${ }^{3}$ Two categories of monolithic CAD/CAM blocks are currently available; resin-based hybrid ceramic blocks and ceramic or glass-ceramic based blocks. The former is easier to mill, easier to finish and polish, less prone to damage with CAM milling burs, less abrasive to opposing teeth and can be easily repaired by composite resins. However, they may experience high wear. ${ }^{4,5}$ Esthetic ceramic blocks, on the other hand, are more wear resistant, more biocompatible and more color stable. ${ }^{6}$

Surface characterization of dental materials plays a pivotal role in deciding the long-term clinical longevity of dental restorations. Surface roughness assessment is considered a cornerstone in evaluating surface characteristics such as; polishing, wear and material degradation. A rough surface of improperly finished and polished or worn restorations may enhance plaque accumulation, increase susceptibility to discoloration and increase abrasion probability of natural antagonist teeth. Moreover, roughened surface may reduce fracture resistance of the materials due to creation and propagation of surface microcracks. ${ }^{6}$

Micro-hardness is another surface property defined as the resistance of the material to permanent surface indentation, scratching or penetration. $\mathrm{Mi}$ cro-hardness is usually correlated to the mechanical strength of the material.?

Dental restorations in the oral cavity suffer from aging which is a process triggered by saliva, chemicals and mechanical stresses due to mastication. Therefore, invitro simulation of the oral environment conditions is important for clinical relevance of the scientific researches. Chewing simulator represents a useful device for studying changes in the surface properties of dental materials during function in the oral cavity. A chewing simulator device should be able to apply force within the masticatory forces range in addition to the application of temperature fluctuations. ${ }^{8}$

Few literatures studied the effect of thermomechanical aging on surface roughness and microhardness of monolithic CAD/CAM blocks. ${ }^{9,} 10$

Therefore, the aim of the current study was to evaluate the changes in surface roughness and micro-hardness of four CAD/CAM materials after thermo-mechanical aging using a chewing simulator accompanied with thermocycling. The null hypothesis is that the thermo-mechanical aging will have no effect either on the surface roughness or the micro-hardness of the four investigated CAD/ CAM materials.

\section{MATERIALS AND METHODS}

\section{Materials}

The four materials tested in the current study are listed in Table 1.

\section{Methods}

\section{Specimens' preparation}

The CAD/CAM blocks of each material were cut under copious amount of water into disks $2 \mathrm{~mm}$ in thickness to obtain 5 disks from each material using a high precision digitally programmed machine (Isomet 5000, Buehler, Lake Buff, IL, USA). Disks were then finished and polished according to mentioned protocols in Table 1. Afterwards, the disks were cleaned for 5 minutes in an ultrasonic 
TABLE (1) Materials tested in the current study.

\begin{tabular}{|c|c|c|c|c|c|c|}
\hline Material & Code & Manufacturer & \multicolumn{2}{|c|}{ Composition } & Polishing of the cut surface & Reference \\
\hline CEREC Blocs & $\mathrm{CB}$ & $\begin{array}{l}\text { Sirona Dental } \\
\text { Systems GmbH }\end{array}$ & \multicolumn{2}{|c|}{$\begin{array}{l}\text { Fine-structured feldspathic ceram- } \\
\text { ic blocks composed of } \mathrm{SiO}_{2}(56-64 \\
\mathrm{wt} \%), \mathrm{Al}_{2} \mathrm{O}_{3}(20-23 \mathrm{wt} \%), \mathrm{Na}_{2} \mathrm{O} \\
(6-9 \mathrm{wt} \%), \mathrm{K}_{2} \mathrm{O}(6-8 \mathrm{wt} \%), \mathrm{CaO} \\
(0.3-0.6 \mathrm{wt} \%), \mathrm{TiO}_{2}(0.0-0.1 \mathrm{wt} \%) .\end{array}$} & $\begin{array}{l}\text { Flexible disks coated with } \mathrm{Al}_{2} \mathrm{O}_{3} \\
\text { and diamond polishing paste }\end{array}$ & 11 \\
\hline Celtra Duo & $\mathrm{CD}$ & $\begin{array}{l}\text { DeguDent } \\
\text { GmbH, } \\
\text { Dentsply, Sirona }\end{array}$ & \multicolumn{2}{|c|}{$\begin{array}{l}\text { Zirconia-reinforced lithium silicate } \\
\text { (ZLS) containing } 10 \mathrm{wt} \% \text { zirconia } \\
\text { in lithium silicate crystallites (500 } \\
-700 \mathrm{~nm})\end{array}$} & $\begin{array}{l}\text { A coarse grit paste was used with } \\
\text { medium and then fine wheels } \\
\text { at speed of } 10,000 \mathrm{rpm} \text { under } \\
\text { a light pressure. Then, a fine } \\
\text { diamond paste was used with a } \\
\text { soft-medium Robinson brush at } \\
\text { speed of } 6,000 \text { rpm under a light } \\
\text { pressure/ } 1 \text { minute each }\end{array}$ & 12,13 \\
\hline \multirow[t]{2}{*}{ CERASMART } & \multirow[t]{2}{*}{$\mathrm{C}$} & \multirow[t]{2}{*}{ GC } & $\begin{array}{l}\text { Polymer } \\
\text { part: }\end{array}$ & $\begin{array}{l}\text { Bis-MEPP, UDMA, } \\
\text { DMA }\end{array}$ & \multirow{2}{*}{$\begin{array}{l}\text { Kuraray Clearfil Twist Dia: } \\
\text { smoothing then polishing Rub- } \\
\text { ber disc/ } 30 \text { seconds each }\end{array}$} & \multirow[t]{2}{*}{$\begin{array}{c}14,15,7 \\
16,17\end{array}$} \\
\hline & & & $\begin{array}{l}\text { Ceramic } \\
\text { part: }\end{array}$ & $\begin{array}{l}71 \mathrm{wt} \% \text { Silica }(20 \\
\mathrm{nm}), \text { Barium glass } \\
(300 \mathrm{~nm})\end{array}$ & & \\
\hline \multirow[t]{2}{*}{ VITA ENAMIC } & \multirow[t]{2}{*}{$\mathrm{E}$} & \multirow[t]{2}{*}{$\begin{array}{l}\text { VITA } \\
\text { Zahnfabrik }\end{array}$} & $\begin{array}{l}\text { Polymer } \\
\text { part: }\end{array}$ & $\begin{array}{l}14 \text { wt } \% \text { UDMA, } \\
\text { TEGDMA }\end{array}$ & \multirow{2}{*}{$\begin{array}{l}\text { Polished using Vita Enamic } \\
\text { polishing set (Vita Zahnfabrik, } \\
\text { Germany) }\end{array}$} & \multirow[t]{2}{*}{18} \\
\hline & & & $\begin{array}{l}\text { Ceramic } \\
\text { part: }\end{array}$ & $\begin{array}{l}86 \mathrm{wt} \% \mathrm{SiO}_{2}, \mathrm{Al}_{2} \mathrm{O}_{3} \\
\mathrm{Na}_{2} \mathrm{O}, \mathrm{K}_{2} \mathrm{O}, \mathrm{B}_{2} \mathrm{O}_{3} \\
\mathrm{ZrO}_{2}, \mathrm{CaO}\end{array}$ & & \\
\hline
\end{tabular}

water bath and stored in distilled water for 24 hours.

\section{Thermo-mechanical Aging:}

Thermo-mechanical aging of specimens was performed using a programmable logic controlled equipment; the newly developed four stations multimodal ROBOTA chewing simulator integrated with thermo-cyclic protocol operated on a servo-motor (Model ACH-09075DC-T, AD-Tech Technology CO., LTD., Germany) that has four chambers with vertical and horizontal movements simultaneously in the thermodynamic condition. All disks were tested against Jackob's chuck as an enamel cusp antagonist fixed in the upper part of each compartment. A weight of $5 \mathrm{~kg}$, which is comparable to $49 \mathrm{~N}$ of chewing force, was exerted. The vertical and horizontal movement distances of

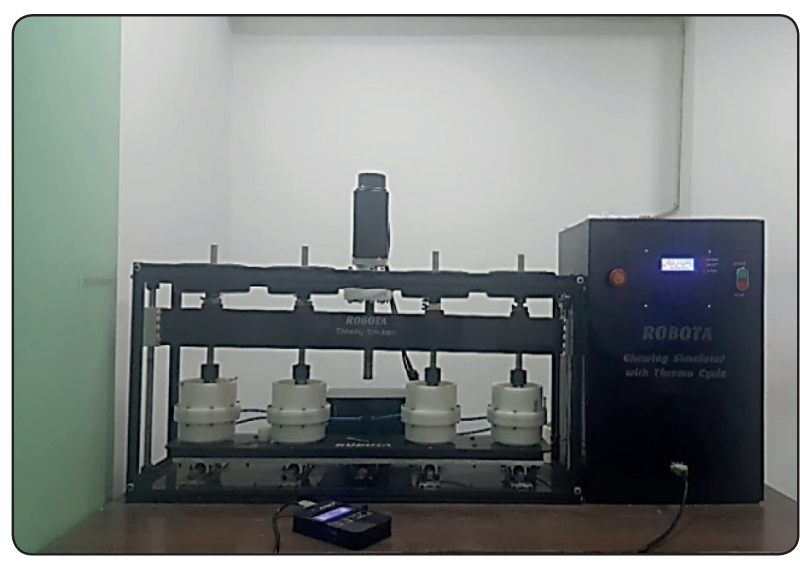

Fig. (1) Robota chewing simulation machine.

the upper part were $1 \mathrm{~mm}$ and $3 \mathrm{~mm}$, respectively, at descending and backward speed of $40 \mathrm{~mm} / \mathrm{s}$. Specimens were subjected to 250,000 times of thermal cycles $\left(5-55^{\circ} \mathrm{C}\right)$ and chewing cycles, at 
frequency of $1.6 \mathrm{~Hz}$, simulating one clinical year according to previous studies. ${ }^{19}$

\section{Surface roughness measurements $\left(\boldsymbol{R}_{a}\right)$}

Working surfaces of disks of all materials were evaluated for their average surface roughness $\left(R_{a}\right)$ pre thermo-mechanical aging (baseline measurement) and post aging at the contact sites in each disk. A measuring technique based on digital image processing technology proposed by Zhongxiang et al. 2009 and Abouelatta 2010 was used. The system consists of two major parts; hardware and software. The hardware consists of: stereomicroscope (Zeiss stereomicroscope, Technival 2), digital camera (Canon, USA), halogen lamp, X, Y bidirectional laboratory bench and a computer. The software transfers the 2D images into numerical values representing the surface roughness values. ${ }^{20,21}$

\section{Scanning electron microscopic (SEM) evaluation}

The working surface of a representative sample from each material was scanned using a SEM (JEOL, JXA-840A, Electron probe microanalyzer, Japan) at magnification power of $500 \mathrm{X}$ to evaluate surface morphology pre and post thermo-mechanical aging at the contact sites.

\section{Surface micro-hardness measurements}

Pre and post micro-hardness measurements were performed at the contact sites on the working surface of each disk. Measurements were done using a standard Vickers diamond pyramid on a hardness testing machine (Vickers; Instron Wolpert, UK). The diamond indenter of the device was used on the working surface of the specimens with a load of $300 \mathrm{~g}$ for 12 seconds and the Vickers hardness number (VHN) was determined. ${ }^{22}$ The average VHN was obtained from the arithmetic mean of three VHN readings pre and post aging. The microhardness values was calculated by substituting in the equation $\mathrm{VHN}=1854.4 \mathrm{~L} / \mathrm{d}^{2}$, where $\mathrm{L}$ is the load in grams and $\mathrm{d}$ is the average diagonal in $\mu \mathrm{m}$.

\section{Statistical analysis}

Data were collected, coded, tabulated and statistically analyzed using the Statistical Package for Social Science (IBM SPSS) version 20 and were presented as means, and standard deviations. The comparison between the four materials regarding quantitative data was done by using one way analysis of variance (ANOVA) test. The results were considered statistically significant at $\mathrm{p}$-value less than or equal to 0.05 ( $\mathrm{p} \leq 0.05)$. For statistically significant results, Bonferroni Post Hoc test was conducted to detect the differences among the groups. In each material, the degree of significance at $p \leq 0.05$ between the pre and post mean results of each test were compared using paired t-test.

\section{RESULTS}

\section{Surface roughness $\left(\mathbf{R}_{\mathrm{a}}\right)$}

The mean $\mathrm{R}_{\mathrm{a}}$ values of pre- and post-measurements of all tested materials are listed in Table 2 and demonstrated in Figure 2. Pre thermo-mechanical aging, all tested CAD/CAM materials showed insignificant difference in surface roughness values. However, post aging, only Cerasmart (C) showed significant higher values $(0.2659 \mu \mathrm{m})$ compared to the other materials. Furthermore, Cerasmart was the only material that showed significant increase in its surface roughness values between pre and post aging values $(0.2527$ and $0.2659 \mu \mathrm{m})$. The surface roughness topography of the four investigated materials pre and post thermo-mechanical aging can be seen in Figure 3.

\section{Scanning electron microscopic (SEM) evaluation}

Scanning electron photomicrographs of the four evaluated materials pre and post aging are demonstrated in Figure 4.

It seemed that all tested materials other than Cerasmart did not show marked changes in their surface topography after thermo-mechanical aging. 
TABLE (2) The mean values (in $\mu \mathrm{m}$ ) and SD of the surface roughness of the tested materials before and after thermo-mechanical aging.

\begin{tabular}{|c|c|c|c|c|c|c|c|c|c|}
\hline & \multicolumn{2}{|c|}{ CB } & \multicolumn{2}{c|}{ CD } & \multicolumn{2}{c|}{ C } & \multicolumn{2}{c|}{ E } \\
\cline { 2 - 11 } & Mean & SD & Mean & SD & Mean & SD & Mean & SD \\
\hline Before & $0.2515^{\mathrm{Aa}}$ & 0.0016 & $0.2533^{\mathrm{Aa}}$ & 0.0018 & $0.2527^{\mathrm{Aa}}$ & 0.0008 & $0.2532^{\mathrm{Aa}}$ & 0.0031 & 0.485 \\
\hline After & $0.2511^{\mathrm{Aa}}$ & 0.0022 & $0.2544^{\mathrm{Aa}}$ & 0.0027 & $0.2659^{\mathrm{Bb}}$ & 0.0002 & $0.2538^{\mathrm{Aa}}$ & 0.0036 & 0.000 \\
\hline Paired t - test & \multicolumn{2}{|c|}{0.180} & \multicolumn{2}{|c|}{0.162} & \multicolumn{2}{|c|}{0.01} & & 0.619 & \\
\hline
\end{tabular}

Values sharing different uppercase letters in the same column and different lowercase letters in the same raw are significantly different at $p \leq 0.05$

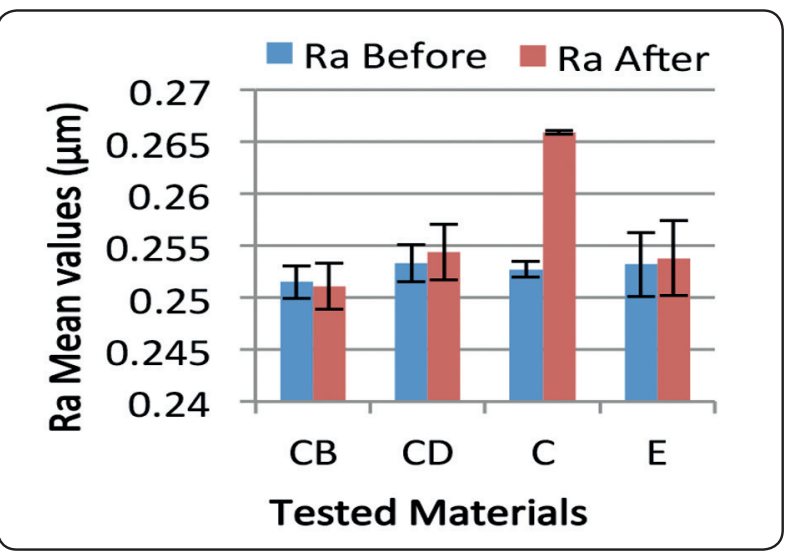

Fig. (2) Histogram showing mean values (in $\mu \mathrm{m}$ ) and SD of the surface roughness of the tested materials before and after thermo-mechanical aging.
Cerasmart showed scratches along the path of chewing simulation direction more than other tested materials.

\section{Surface micro-hardness}

The mean Vickers micro-hardness values are listed in Table 3 and illustrated in Figure 5. Before thermo-mechanical aging, all materials showed significant difference in surface micro-hardness values. On the other hand, after aging, all materials showed insignificant differences in their surface micro-hardness values. Additionally, all materials showed insignificant change in micro-hardness values between pre and post aging.

TABLE (3) The mean values and SD of the Vickers hardness of the tested materials before and after thermomechanical aging.

\begin{tabular}{|c|c|c|c|c|c|c|c|c|c|}
\hline & \multicolumn{2}{|c|}{$\mathrm{CB}$} & \multicolumn{2}{|c|}{$\mathrm{CD}$} & \multicolumn{2}{|c|}{$\mathrm{C}$} & \multicolumn{2}{|c|}{$\mathrm{E}$} & \multirow{2}{*}{$P$ Value } \\
\hline & Mean & SD & Mean & SD & Mean & SD & Mean & SD & \\
\hline Before & $382.01^{\mathrm{Ac}}$ & 22.49 & $496.25^{\mathrm{Ad}}$ & 11.64 & $184.62^{\mathrm{Aa}}$ & 31.93 & $248.19^{\mathrm{Ab}}$ & 12.05 & 0.000 \\
\hline After & $358.06^{\mathrm{Aa}}$ & 50.50 & $339.17^{\mathrm{Aa}}$ & 13.93 & $359.20^{\mathrm{Aa}}$ & 14.94 & $379.04^{\mathrm{Aa}}$ & 34.23 & 0.315 \\
\hline Paired $\mathrm{t}$ - test & \multicolumn{2}{|c|}{0.065} & \multicolumn{2}{|c|}{0.816} & \multicolumn{2}{|c|}{0.16} & \multicolumn{2}{|c|}{0.164} & \\
\hline
\end{tabular}

Values sharing different uppercase letters in the same column and different lowercase letters in the same raw are significantly different at $p \leq 0.05$ 


\section{Pre}
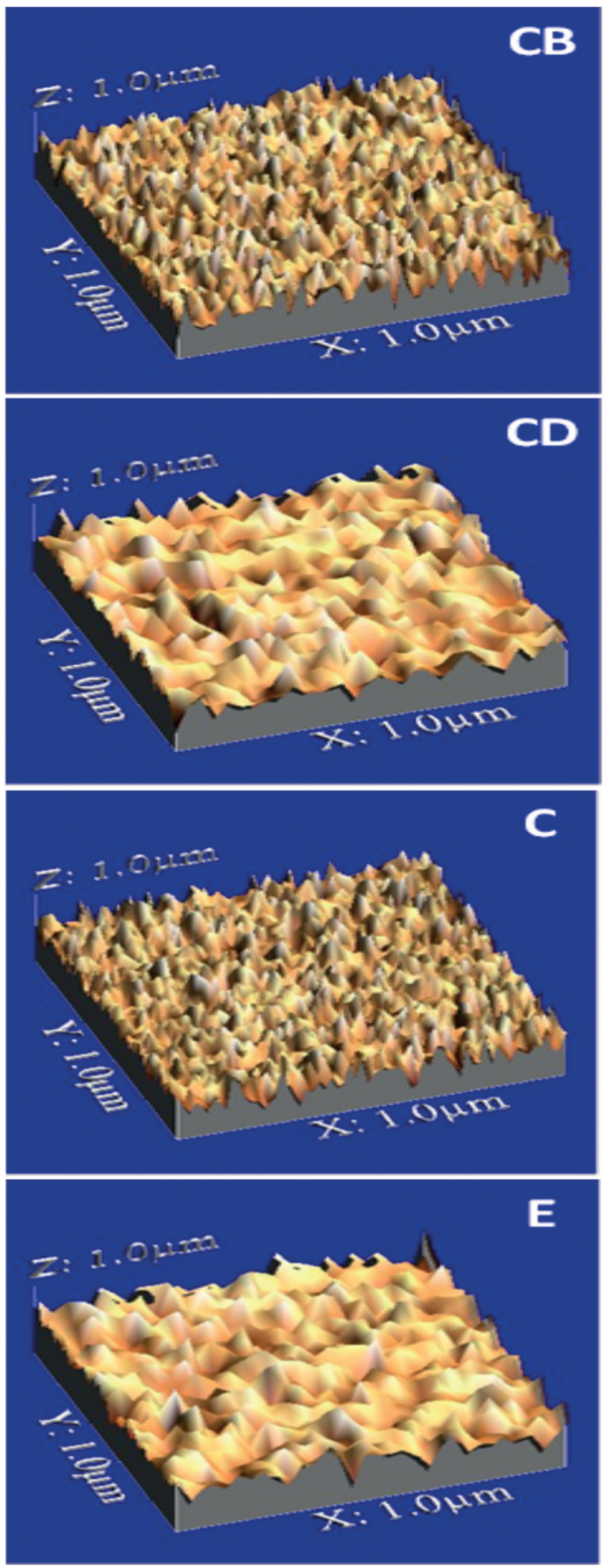

Post

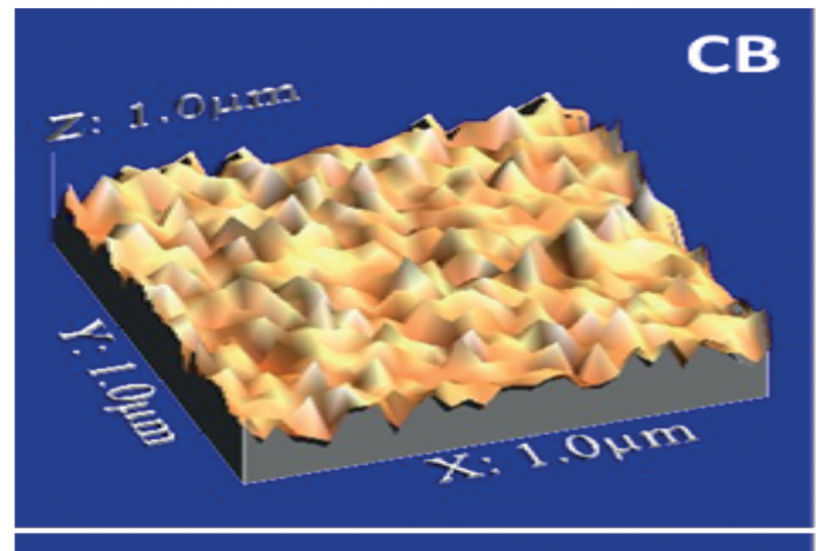

CD
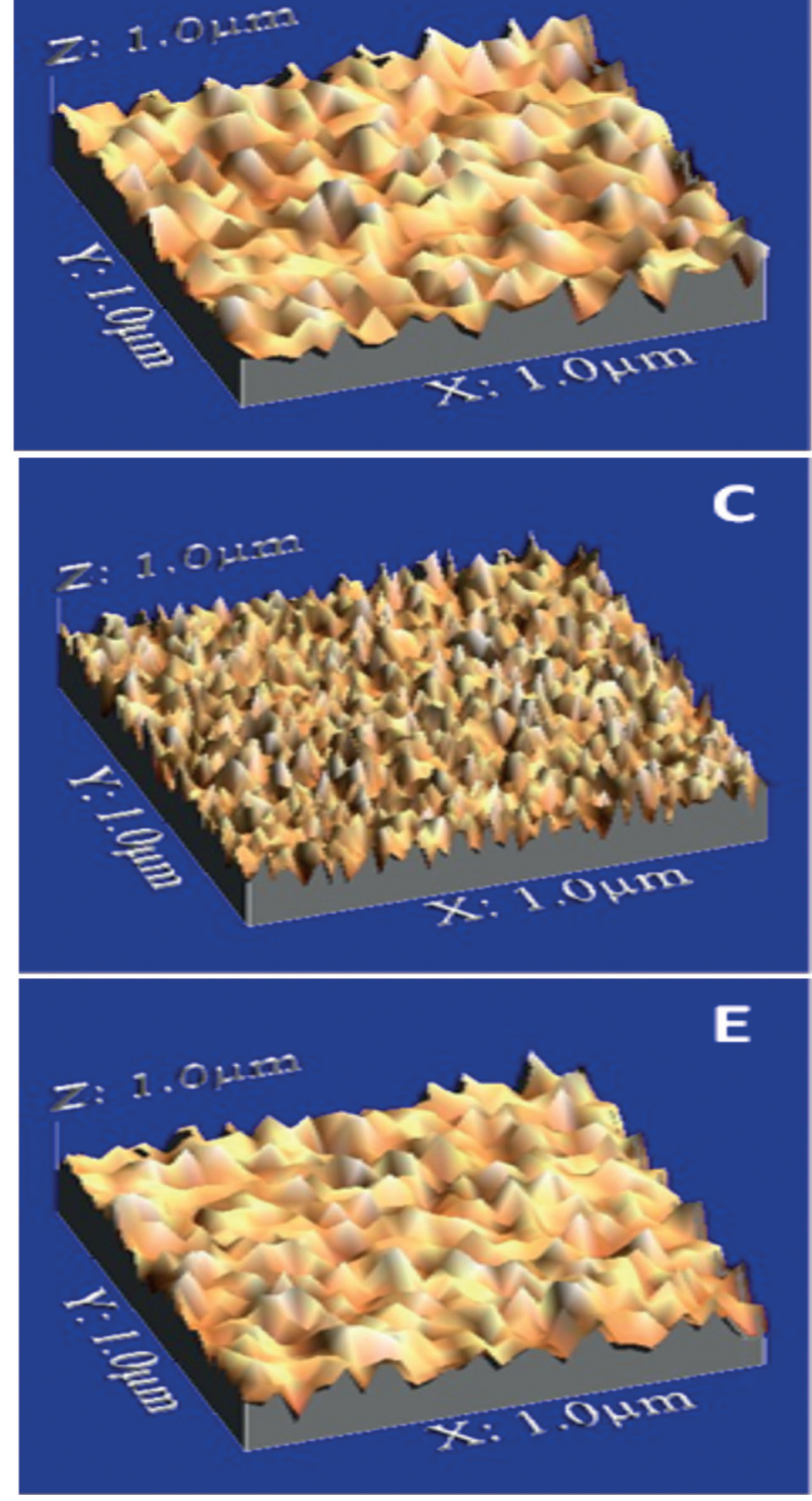

Fig. (3) Surface roughness topography of the four tested materials pre and post thermo-mechanical aging. 


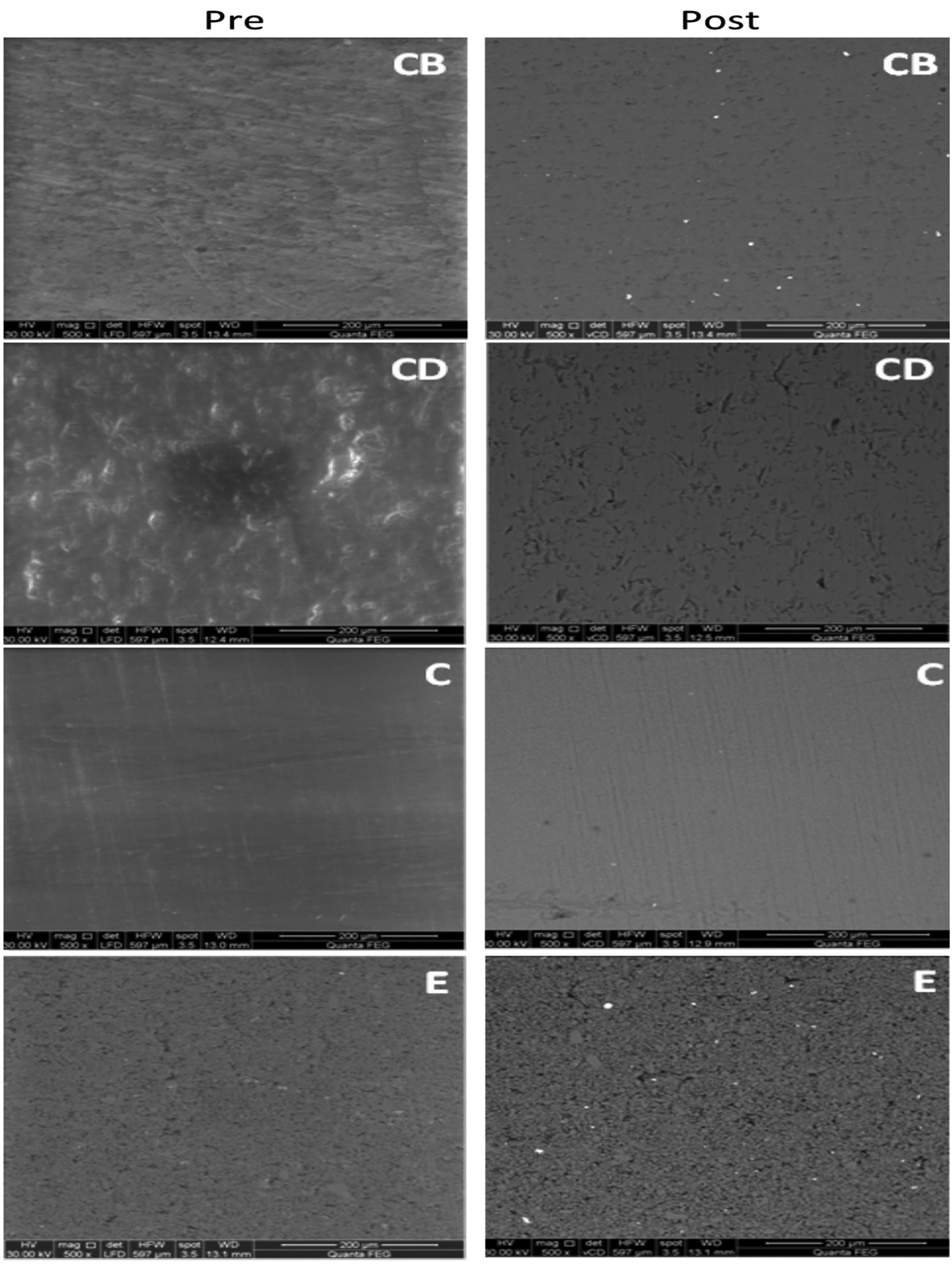

Fig. (4) SEM photomicrographs of the four tested materials pre and post thermo-mechanical aging (500X). 


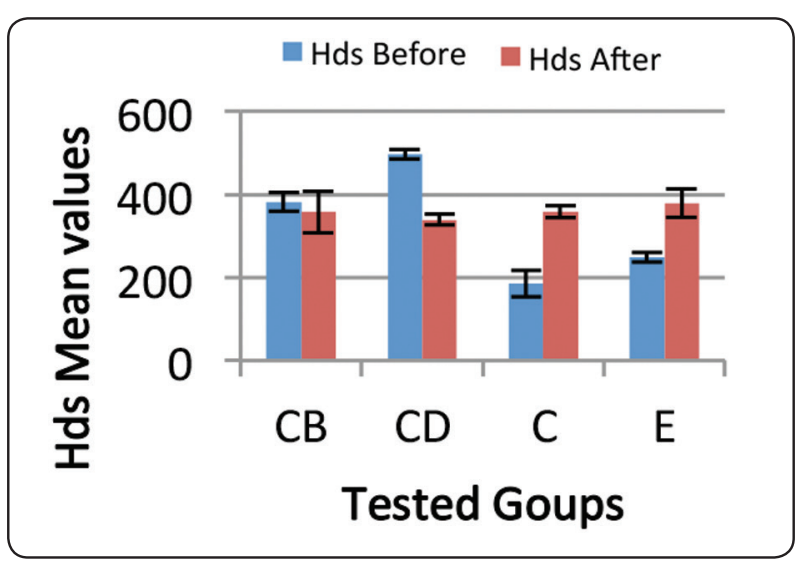

Figure 5: Histogram showing mean values and SD of the Vickers hardness of the tested materials before and after thermo-mechanical aging.

\section{DISCUSSION}

Dental materials inserted in the oral cavity are subjected to various thermal fluctuations, chemical changes and mechanical stresses which might affect their surface qualities, which in turn would affect the longevity of de ntal restorations.

\section{Surface roughness}

According to Jones et al ${ }^{23}$ the $\mathrm{R}_{\mathrm{a}}$ values up to $0.28 \mu \mathrm{m}$ could be clinically accepted. Therefore, the results of surface roughness for all the tested materials were in the acceptable clinical range.

The null hypothesis for the effect of thermomechanical aging on surface roughness $\left(R_{a}\right)$ of the tested materials was partially rejected for Cerasmart only. Cerasmart showed a significant increase in roughness from $0.2527 \mu \mathrm{m}$ to $0.2659 \mu \mathrm{m}$. This increase in surface roughness of Cerasmart might be attributed mainly to water sorption. Water would penetrate into the resin matrix through two mechanisms; free volume mechanism and interaction mechanism. Water diffuses by the former mechanism just occupies the free volume between the polymer chains and the nanopores created during polymerization and is referred to as unbounded water. In the second mechanism, water diffuses through the material binding successively to the polymer hydrophilic groups via hydrogen bonds and is referred to as bounded water. ${ }^{24}$

In addition, the weight percentage of the resin matrix play an important role in the amount of water sorption in the hybrid ceramics. The lower the weight percent of ceramic fillers, the higher is the weight percent of the polymeric matrix, and the higher is the water sorption.25,8 The percent of fillers in Cerasmart is only $71 \mathrm{wt} \%$, compared to $86 \mathrm{wt} \%$ in Vita Enamic. This greater amount of polymeric matrix in Cerasmart than in Vita Enamic could be also a direct cause for the greater water sorption of Cerasmart. Moreover, water penetration into the surface of Cerasmart blocks which are classified as a hybrid nano-ceramic blocks, could be attributed to the hydrophilicity of the resin matrix and/or hydrolytic instability of the interfacial coupling agent between filler and resin matrix. ${ }^{26}$

This goes in agreement with Lauvahutanon et al, ${ }^{27}$ who studied the effect of immersion of Cerasmart, Vita Enamic and feldspar ceramic blocks in 2 different liquids. The results showed that the water sorption of Cerasmart was significantly higher than in Enamic and that Feldspar ceramic blocks showed almost zero water sorption. The study attributed the water sorption in Cerasmart to the presence of nano sized fillers with a high total surface area making salinization of these nano sized fillers a difficult process. Hence, Cerasmart was more prone to water sorption.

The absorbed water results in expansion and plasticization of the resin matrix and diffuses into the interface between the ceramic and polymeric phases. This might result in the hydrolysis of the silane coupling agent with eventual loss of surface fillers. Moreover, it might result in creating microcracks in the surface leading to increased surface roughness of Cerasmart compared to other tested materials. ${ }^{26,28}$ 
Once more, the results of this study came in agreement with the results of Koizumi et al. ${ }^{29}$ which found that after 20,000 brushing cycles, there was a significant increase in the roughness parameter $\mathrm{R}_{\mathrm{a}}$ of Cerasmart than that of Enamic and feldspathic porcelain blocks. The study attributed this significant increase to the much higher hardness of the filler particles than the surrounding matrix resin which was readily worn.

On the other hand, Tekçe et al, ${ }^{6}$ after subjecting Vita Enamic and Cerasmart CAD/CAM materials to aging procedures, found significant reduction in $\mathrm{R}_{\mathrm{a}}$ values of Vita Enamic with no effect on the Cerasmart samples. This difference would be due to their adoption of only 5000 thermocycles without frictional movements of the chewing simulations used in the current study.

\section{Surface micro-hardness}

The surface micro-hardness testing is useful to assess surface characteristics in relation to wear resistance. ${ }^{30}$ Vickers hardness test is a common method to test hardness. ${ }^{29,30}$

Regarding micro-hardness the null hypothesis for the effect of thermo-mechanical aging on surface micro-hardness of the tested materials was accepted.

The results showed significant difference between all tested materials before subjecting them to thermo-mechanical aging. The micro-hardness results before thermo-mechanical aging were in the ascending order of Cerasmart $<$ Enamic $<$ Cerec Blocks $<$ Celtra Duo. This order came in agreement with those of Koizumi et al.$^{29}$ who found significant difference in micro-hardness of ceramic disks with the feldspathic porcelain was the highest and Cerasmart was the lowest. Their study suggested that micro-hardness of the hybrid ceramic blocks is considerably affected not only by inorganic filler contents but also, they can be affected by filler form, size and the organic polymeric matrix.

Rybarek ${ }^{31}$ suggested that the presence of TEGDMA would markedly increase the hardness of the resin matrix as it has very high concentration of double bonds as well as high degree of conversion compared to UDMA. This would result in highest crosslinking and forming the tightest networks. This might be another reason for increased hardness of Vita Enamic compared to Cerasmart as the resin matrix of Vita Enamic contains TEGDMA, while the resin matrix of Cerasmart is devoid of this component.

Furthermore, Rybarek and Jurczyk ${ }^{32}$ evaluated the hardness of UDMA/TEGDMA copolymer which is the typical copolymer forming the Vita Enamic matrix. They found UDMA/TEGDMA copolymer to be one of the hardest copolymer investigated in their study.

The results of the current study is in agreement with study of Lawson et al $^{7}$ which found Cerasmart much softer than Celtra Duo, while Vita Enamic was intermediate with significant differences among all those materials. In another study, micro-hardness of feldspathic porcelain, resin nano ceramic and dual network ceramic (Vita Enamic) disks was also tested. Same ranking as the current study was also noticed and the differences in that ranking were also statistically significant. ${ }^{22}$

After thermo-mechanical aging, all tested materials showed insignificant difference in their surface micro-hardness values, while, the micro-hardness of each material did not change significantly between pre and post aging. The changes in micro-hardness values after aging so that hybrid ceramic materials matched the micro-hardness values of ceramic materials could be due to the worn down resin matrix exposing inorganic fillers. Those exposed fillers could be hard enough to raise the micro-hardness values making the difference between those materials and ceramic ones insignificant.

Finally, the low micro-hardness values of Cerasmart due to the lower filler content could explain its significant increase in $\mathrm{R}_{\mathrm{a}}$ values after aging based on the direct relation between surface micro-hardness and wear. ${ }^{29,30,33}$ 


\section{CONCLUSIONS}

Within the limitations of the current study, it can be concluded that:

1. Although within the clinical acceptable range, the surface roughness of Cerasmart was significantly affected by the thermo-mechanical aging.

2. The chemical composition is the most important parameter affecting the surface roughness and micro-hardness of CAD/CAM ceramic and hybrid ceramic materials.

\section{REFERENCES}

1. N. D. Ruse and M. J. Sadoun, "Resin-composite blocks for dental CAD/CAM applications," J. Dent. Res., vol. 93, no. 12, pp. 1232-1234, Dec. 2014.

2. K. Baroudi and S. N. Ibraheem, "Assessment of Chairside Computer-Aided Design and Computer-Aided Manufacturing Restorations: A Review of the Literature," J Int Oral Health, vol. 7, no. 4, pp. 96-104, Apr. 2015.

3. L. H. da Silva, E. de Lima, R. B. de P. Miranda, S. S. Favero, U. Lohbauer, and P. F. Cesar, "Dental ceramics: a review of new materials and processing methods," Brazilian Oral Research, vol. 31, no. suppl 1, Aug. 2017.

4. N. D. Ruse and M. J. Sadoun, "Resin-composite Blocks for Dental CAD/CAM Applications," Journal of Dental Research, vol. 93, no. 12, pp. 1232-1234, Dec. 2014.

5. G. Chirumamilla, C. E. Goldstein, and N. C. Lawson, "A 2-year Retrospective Clinical study of Enamic Crowns Performed in a Private Practice Setting: Clinical Study of Enamic Crowns Performed in a Private Practice Setting," Journal of Esthetic and Restorative Dentistry, vol. 28, no. 4, pp. 231-237, Jul. 2016.

6. N. Tekçe, S. Fidan, S. Tuncer, D. Kara, and M. Demirci, The effect of glazing and aging on the surface properties of CAD/CAM resin blocks, The Journal of Advanced Prosthodontics, vol. 10, no. 1, p. 50, 2018.

7. H. Koizumi, O. Saiki, H. Nogawa, H. Hiraba, T. Okazaki, and H. Matsumura, "Surface roughness and gloss of current CAD/CAM resin composites before and after toothbrush abrasion,” Dental Materials Journal, vol. 34, no. 6 , pp. 881-887, 2015 .
8. R. Z. Alshali, N. A. Salim, J. D. Satterthwaite, and N. Silikas, "Long-term sorption and solubility of bulk-fill and conventional resin-composites in water and artificial saliva," Journal of Dentistry, vol. 43, no. 12, pp. 15111518, Dec. 2015.

9. N. C. Lawson, R. Bansal, and J. O. Burgess, "Wear, strength, modulus and hardness of CAD/CAM restorative materials," Dental Materials, vol. 32, no. 11, pp. e275e283, Nov. 2016.

10. N. Sonmez, P. Gultekin, V. Turp, G. Akgungor, D. Sen, and E. Mijiritsky, "Evaluation of five CAD/CAM materials by microstructural characterization and mechanical tests: a comparative in vitro study," BMC Oral Health, vol. 18, no. 1, Dec. 2018

11. CEREC Blocs, Sirona Dental Systems GmbH, 2010.

12. Celtra ${ }^{\circledR}$ Duo, Brochure for the dental laboratory, The Dental Solution Company, Dentsply Sirona, 2017.

13. Celtra ${ }^{\circledR}$ Duo, Guidelines for processing Celtra Duo, The Dental Solution Company, Dentsply Sirona, 2018

14. CERASMART ${ }^{\mathrm{TM}}$, GCERAMICA, 2014

15. CERASMART ${ }^{\mathrm{TM}}$ from GC, GCERAMICA, 2015

16. A. Goujat et al., "Mechanical properties and internal fit of 4 CAD-CAM block materials," The Journal of Prosthetic Dentistry, vol. 119, no. 3, pp. 384-389, Mar. 2018.

17. N. Tekçe, S. Fidan, S. Tuncer, D. Kara, and M. Demirci, "The effect of glazing and aging on the surface properties of CAD/CAM resin blocks," The Journal of Advanced Prosthodontics, vol. 10, no. 1, p. 50, 2018.

18. VITA ENAMIC ${ }^{\circledR}$, Technical and scientific documentation, VITA Zahnfabrik H., 2016.

19. N. Nawafleh, M. Hatamleh, S. Elshiyab, and F. Mack, "Lithium Disilicate Restorations Fatigue Testing Parameters: A Systematic Review: Lithium Disilicate Fatigue Testing Parameters," Journal of Prosthodontics, vol. 25, no. 2, pp. 116-126, Feb. 2016

20. H. Zhongxiang, Z. Lei, T. Jiaxu, M. Xuehong, and S. Xiaojun, "Evaluation of three-dimensional surface roughness parameters based on digital image processing," The International Journal of Advanced Manufacturing Technology, vol. 40, no. 3-4, pp. 342-348, Jan. 2009.

21. O. B. Abouelatta, "3D Surface Roughness Measure- ment Using a Light Sectioning Vision System, Proc. World Congr", Eng. I. 2010. 
22. N. Şen, B. Tuncelli, and G. Göller, "Surface deterioration of monolithic CAD/CAM restorative materials after artificial abrasive toothbrushing," The Journal of Advanced Prosthodontics, vol. 10, no. 4, p. 271, 2018.

23. C. S. Jones, R. W. Billington, and G. J. Pearson, "The in vivo perception of roughness of restorations," British Dental Journal, vol. 196, no. 1, pp. 42-45, Jan. 2004.

24. K. Bociong et al., "The Influence of Water Sorption of Dental Light-Cured Composites on Shrinkage Stress," Materials, vol. 10, no. 10, p. 1142, Sep. 2017.

25. T. Misilli and N. Gönülol, "Water sorption and solubility of bulk-fill composites polymerized with a third generation LED LCU," Brazilian Oral Research, vol. 31, no. 0, Oct. 2017.

26. T. Hirasawa, S. Hirano, I. Harashima, S. Hirabayashi, and R. Mori, "[Mechanical and dimensional properties of thermosetting resins for crown (author's transl)]," Shika Rikogaku Zasshi, vol. 20, no. 52, pp. 209-216, Oct. 1979.

27. S. Lauvahutanon et al., "Discoloration of various CAD/ CAM blocks after immersion in coffee," Restorative Dentistry \& Endodontics, vol. 42, no. 1, p. 9, 2017.

28. M. Toledano, R. Osorio, E. Osorio, V. Fuentes, C. Prati, and F. García-Godoy, "Sorption and solubility of resin- based restorative dental materials," Journal of Dentistry, vol. 31, no. 1, pp. 43-50, Jan. 2003.

29. H. Koizumi, O. Saiki, H. Nogawa, H. Hiraba, T. Okazaki, and H. Matsumura, "Surface roughness and gloss of current $\mathrm{CAD} / \mathrm{CAM}$ resin composites before and after toothbrush abrasion," Dental Materials Journal, vol. 34, no. 6,pp. 881-887, 2015.

30. M. Murakami, "Surface properties of an indirect composite polymerized with five laboratory light polymerization systems," J Oral Sci, vol. 51, no. 2, pp. 215-221, Jun. 2009.

31. I. M. Barszczewska-Rybarek, "Structure-property relationships in dimethacrylate networks based on Bis-GMA, UDMA and TEGDMA," Dental Materials, vol. 25, no. 9, pp. 1082-1089, Sep. 2009.

32. I. Barszczewska-Rybarek and S. Jurczyk, "Comparative Study of Structure-Property Relationships in Polymer Networks Based on Bis-GMA, TEGDMA and Various Urethane-Dimethacrylates," Materials, vol. 8, no. 3, pp. 1230-1248, Mar. 2015.

33. M. N. Mandikos, G. P. McGivney, E. Davis, P. J. Bush, and J. M. Carter, "A comparison of the wear resistance and hardness of indirect composite resins," J Prosthet Dent, vol. 85, no. 4, pp. 386-395, Apr. 2001. 